\title{
Pleural infection on the increase but with a better evidence base to inform clinical care
}

\author{
Najib M Rahman, ${ }^{1}$ Nick A Maskell ${ }^{2}$
}

Infection of the pleural space is an ancient disease, with the earliest recorded description more than 5000 years ago, ${ }^{1}$ and the first consistent description of its manifestations and treatment credited to the father of modern medicine, Hippocrates. ${ }^{2}$ Open thoracic drainage, with its associated high mortality, remained the standard treatment for pleural infection until the influenza pandemic of 1919, when closed tube drainage techniques described in the 19th century $^{3} 4$ were used, and significantly reduced the associated mortality. ${ }^{5}$ The treatment principles described almost 100 years ago remain to this day in the treatment of pleural infection. ${ }^{6}$

For many years, pleural infection was considered to be a result of 'pneumonia gone bad' with fluid leaking out of the infected lung parenchyma resulting in an infected fluid collection which was poorly accessible to the immune system. The term 'parapneumonic effusion' exemplifies this possible aetiology. However, recent studies ${ }^{7}$ describing the microbiology associated with pleural infection suggest that this may be a too simplistic understanding of the pathological processes occurring. There are markedly different bacteriological patterns in pleural infection ${ }^{7}$ compared with pneumonia, suggesting microbiologically distinct diseases. Nonetheless, the more widespread use of early antibiotic therapy for pneumonia may be expected to result in decreasing rates of pleural infection, if all pleural infection is simply a complication of pneumonia not treated early enough.

Recent evidence suggests that this may not be the case, and it may be time to rethink our understanding of the

\footnotetext{
${ }^{1}$ Oxford Respiratory Trials Unit, Oxford Centre for Respiratory Medicine, Churchill Hospital, University of Oxford, Oxford, UK; ${ }^{2}$ North Bristol Lung Centre, Southmead Hospital, Bristol University, Bristol, UK

Correspondence to Najib M Rahman, Oxford Centre for Respiratory Medicine, Churchill Hospital, University of Oxford, Old Road, Headington, Oxford OX3 7LJ, UK; naj_rahman@yahoo.co.uk
}

development of pleural infection. The study by Grijalva et $a l^{8}$ in this issue of Thorax provides significant new data to a number of studies from the last 4 years $^{9-11}$ demonstrating increasing incidence of pleural infection in both the adult and paediatric populations (see page 663). While a number of previous studies may be subject to selection bias, Grijalva et al employed a robust study design to minimise this, using a large national database of a wide range of hospital settings, an unbiased sampling strategy and wide diagnostic and procedural criteria. In addition, the authors stratified incidence changes by age (including paediatric and adult populations) and perhaps most interestingly by organism.

The authors report a significant (twofold) increase in the incidence of pleural infection-as expected, the incidence was highest in the oldest age strata (>65 years), but all age groups experienced a roughly twofold increase over the study period. Perhaps most interestingly, while rates of pneumococcal empyema appeared to remain stable in both adult and paediatric populations, the incidence of streptococcal and especially staphylococcal empyema showed a significant increase over the study period.

This study thus provides further evidence of the increasing burden of disease associated with pleural infection, and begins to pose intriguing questions as to the aetiology of this consistently reported increase. Within the paediatric sphere, there is continued debate as to the role of the heptavalent pneumococcal vaccine as a potential causative factor in this increase, ${ }^{12}{ }^{13}$ while the Grijalva et al study suggests a changing microbiological pattern associated with increased incidence, perhaps favouring more resistant organisms that have been associated with a poorer prognosis in previous studies. ${ }^{7}$

The potentially changing microbiological face of pleural infection highlights the importance of swift and accurate microbiological diagnosis. The clinician treating pleural infection is at a particular disadvantage-previous studies demonstrate that around $40 \%$ of pleural infection will remain microbiologically obscure despite standard pleural fluid culture. ${ }^{7}$ Organisms resistant to standard pneumonia antibiotic regimens are common, and thus a significant proportion of patients will require empirical broadspectrum antibiotics for the duration of their treatment, ${ }^{6}$ with the well-known attendant risks. Although several studies have assessed the use of bacterial genetic techniques to increase diagnostic yield, ${ }^{74-16}$ these techniques are far from proven in the clinical context and currently confined to research centres.

The study by Menzies et al (see page 658) provides the first comparative, prospective evidence of increased microbiological diagnostic yield in pleural infection using a widely available clinical test (the BACTEC blood culture bottle system, Becton, Dickinson U.K. Limited, Oxford, UK). ${ }^{17}$ Addition of blood culture bottle inoculated pleural fluid to standard pleural fluid culture increased microbiological diagnostic yield by $21 \%$, and in a small proportion of cases (4\%) where standard culture was positive, blood culture bottle inoculation suggested the presence of organisms which would alter antibiotic management. These findings are further supported by the lack of false positivity in control samples. Intriguingly, 'standard' culture was positive where the pleural fluid in blood culture bottle was negative in $29 \%$ of cases, suggesting potential organism preference for certain growth medium.

Complex bacterial genetic techniques merit further clinical investigation and may become an important diagnostic modality in the future. However, the study by Menzies et $a l^{17}$ demonstrates a significant increase in diagnostic yield using a widely available and relatively inexpensive technique, suggesting that inoculation of pleural fluid into blood culture bottles as an addition to standard pleural fluid culture should be added to standard practice today.

There is therefore compelling evidence that pleural infection is on the increase, and the suggestion that organisms associated with a poorer prognosis may be responsible. Although the precise reasons for this trend remain obscure, it is clear that pleural infection will continue to be an important clinical entity in everyday respiratory practice. Clinical studies, such as the two studies highlighted in this editorial, are vital in advancing our understanding of disease pattern and process, and improving delivery of care. 
We would like to end this editorial with a tribute to Professor Robert J O Davies who unexpectedly died recently, and whose career was exemplified by such studies. He was well known around the world as a leading authority in pleural diseases and made significant contributions to the field, with a strong and lasting impact on the clinical care of patients with pleural disease. Professor Davies was instrumental in changing the image of pleural disease from a stagnant and ignored field to a vibrant subspecialty interest in the UK and beyond. He was passionate in promoting evidence-based care for pleural disease patients, and to this end set up a Respiratory Clinical Trials Unit at the Churchill Hospital in Oxford, from where he coordinated several landmark multicentre studies addressing key clinical questions in pleural infection, malignant pleural effusion and sleep medicine. His enthusiasm and drive generated a successful network of collaborating departments throughout the UK and he chaired the highly influential 2003 British Thoracic Society guidelines for the management of pleural disease, further contributing to his international reputation.

His contribution to pleural infection is perhaps best summed up by the most recent BTS Pleural Disease Guidelines, where his published original papers contributed to 5 of the $16(31 \%)$ grade $A$ or $\mathrm{B}$ recommendations in the pleural infection section. He will be greatly missed personally and professionally.

Acknowledgements We thank Professor John Stradling (Oxford, UK) for his help in writing the obituary.

Provenance and peer review Commissioned; not externally peer reviewed.

Published Online First 15 June 2011

Thorax 2011;66:649-650.

doi:10.1136/thx.2011.161315

\section{REFERENCES}

1. Breasted JH. The Edwin Smith Surgical Papyrus. Chicago, IL: University of Chicago Press, 1980.

2. Hippocrates. Aphorisms 44. In: Adams LB, ed. The Genuine Works of Hippocrates: The Classics of Surgery. Birmingham, AL: Gryphon Editions, 1985:768-71.

3. Hewitt C. Drainage for empyema. BMJ 1876;1:317.

4. Meyer JA. Gotthard Bulau and closed water-seal drainage for empyema, 1875-1891. Ann Thorac Surg 1989;48:597-9.

5. Stone WJ. The management of postpneumonic empyema based on 310 cases. Am J Med Sci 1919;158:1-29.

6. Davies HE, Davies RJ, Davies CW. Management of pleural infection in adults: British Thoracic Society Pleural Disease Guideline 2010. Thorax 2010;65(Suppl 2):ii41-53.

7. Maskell NA, Batt S, Hedley EL, et al. The bacteriology of pleural infection by genetic and standard methods and its mortality significance. Am J Respir Crit Care Med 2006;174:817-23.
8. Grijalva CG, Zhu Y, Nuorti JP, et al. The emergence of parapneumonic empyema in the United States. Thorax 2011;66:663-8.

9. Finley C, Clifton J, Fitzgerald JM, et al. Empyema: an increasing concern in Canada. Can Respir $J$ 2008;15:85-9.

10. Farjah F, Symons RG, Krishnadasan B, et al Management of pleural space infections: a population-based analysis. J Thorac Cardiovasc Surg 2007;133:346-51.

11. Roxburgh CS, Youngson GG. Childhood empyema in North-East Scotland over the past 15 years. Scott Med J 2007:52:25-7.

12. Munoz-Almagro C, Jordan I, Gene A, et al. Emergence of invasive pneumococcal disease caused by nonvaccine serotypes in the era of 7 -valent conjugate vaccine. Clin Infect Dis 2008;46:174-82.

13. Bekri H, Cohen R, Varon E, et al. Streptococcus pneumoniae serotypes involved in children with pleural empyemas in France. Arch Pediatr 2007; 14:239-43.

14. Saglani S, Harris KA, Wallis C, et al. Empyema: the use of broad range $16 \mathrm{~S}$ rDNA PCR for pathogen detection. Arch Dis Child 2005; 90:70-3.

15. Menezes-Martins LF, Menezes-Martins JJ, Michaelsen VS, et al. Diagnosis of parapneumonic pleural effusion by polymerase chain reaction in children. $J$ Pediatr Surg 2005; 40:1106-10.

16. Le Monnier A, Carbonnelle E, Zahar JR, et al. Microbiological diagnosis of empyema in children: comparative evaluations by culture, polymerase chain reaction, and pneumococcal antigen detection in pleural fluids. Clin Infect Dis 2006;42:1135-40.

17. Menzies SM, Rahman NM, Wrightson JM, et al. Blood culture bottle culture of pleural fluid in pleural infection. Thorax 2011;66:658-62.

\section{Thorax alerts}

Sign up for our electronic table of contents alerts and you will never miss new issues of Thorax when published online. Stay ahead and up to date by visiting thorax.bmj.com. 